\title{
Ontodomo: um modelo de base de conhecimentos terminológicos
}

Joaquim Rodrigues Bento ${ }^{1}$

RESUMO: Neste artigo, apresentamos alguns resultados de nossa tese de Doutoramento, na qual propusemo-nos construir um modelo de base de conbecimentos terminológicos (BCT) a partir de um corpus especializado (Domo corpus). O objectivo principal deste estudo centrou-se na definição dos fundamentos teórico-metodológicos, assim como na experimentação de diferentes técnicas e sistemas de tratamento automático (e assistido) da língua, para a aquisição, a partir do corpus, da terminologia e das relações conceptuais necessárias à estruturação terminológica e modelização do corpus e do domínio. Nestas breves páginas, pretendemos apenas mostrar as características principais de nosso modelo BCT, implementado informaticamente no sistema OntoDomo.

Palavras-chave: Lexicologia; terminologia; terminologia descriptiva; estruturação terminológica; Processamento de Linguagem Natural (PLN); Base de Conhecimentos Terminológicos (BCT); ontologia; recuperação de informação (RI).

ABSTRACT: In this article, we present some results of our Ph.D. thesis research in which we proposed to build a model of a Terminological Knowledge Base (TKB) based on a specialized corpus (Domo corpus). The main purpose of our research was the definition of the theoretical and methodological foundations, as well the experimentation of different Natural Language Processing (NLP) tools and techniques for the acquisition of terms and conceptual relationships, which are necessary both for the terminological structuring, the corpus and the domain modelling. In these few pages, we intend only to show the main features of our TBK model, implemented in an integrated computer system - OntoDomo.

Keywords: Lexicology; terminology; descriptive terminology; terminology structuration; Natural Language Processing (NLP); Terminological Knowledge Base (TKB); ontology; information retrieval (RI).

Instituto Politécnico de Viseu, Portugal. E-mail: bentoesev@esev.ipv.pt. 
1. Nota introdutória

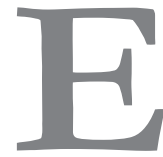

ste projecto, realizado no âmbito da investigação de Doutoramento (Bento, 2007) incidiu, como o nome pretende sugerir, no domínio de especialização tecnológico de edifícios inteligentes e domótica.

Nesta investigação, propusemo-nos construir um modelo de base de conbecimentos terminológicos (BCT), a partir de um corpus especializado, constituído por um conjunto de teses e artigos académicos, produzidos no âmbito da investigação de nível superior, em Portugal.

O objectivo deste estudo centrou-se na definição dos fundamentos teórico-metodológicos e na experimentação de diferentes técnicas e sistemas de tratamento automático (e assistido) da língua, para a aquisição, a partir do corpus, da terminologia e das relações conceptuais necessárias à estruturação terminológica e modelização do corpus e do domínio.

Remetendo toda a fundamentação para a obra supracitada, neste artigo, apresentamos a implementação informática, o sistema OntoDomo, desenvolvido no Centro de Linguística da Universidade Nova de Lisboa, "linha 2 - Lexicologia, Lexicografia e Terminologia", sob a Direcção da Professora Doutora Teresa Lino, com os seguintes objectivos aplicacionais:

- A recuperação de informação e consulta assistida de documentação especializada.

- A navegação na estrutura conceptual do domínio (núcleo ontológico da BCT) e a visualização dos grafos conceptuais gerados pelo sistema;

- A consulta da informação terminográfica considerada relevante.

2. O modelo de dados ontodomo

A definição de um modelo de dados serve de orientação na análise do corpus e na aquisição dos dados conceptuais e terminológicos (cf. Condamines, 2003). Assim, a primeira tarefa consiste em definir o que é relevante adquirir no corpus. Neste estudo, adoptou-se o modelo de dados explicitado na figura 1, explanado através do conceito de detector de gás. 


\begin{tabular}{|c|c|c|c|}
\hline \multicolumn{2}{|c|}{ CONCEITO } & 82.00 & DETECTOR DE GÁS \\
\hline \multicolumn{2}{|c|}{ Domínio } & 003 & protecção contra incêndio \\
\hline Relação genérica & is_a & 001 & detector \\
\hline \multirow[t]{2}{*}{ Relações partitivas } & part_of_c & 002 & sistema domótico \\
\hline & part_of_ph & 003 & \\
\hline \multirow[t]{2}{*}{ Relações funcionais } & used_for & 004 & detecção de fugas de gás \\
\hline & has_purpose & 005 & protecção \\
\hline \multirow{3}{*}{$\begin{array}{l}\text { Relações específicas } \\
\text { (exemplos) }\end{array}$} & entrada & 006 & presença de gás combustível \\
\hline & saída & 007 & sinal eléctrico binário $(0 / 1)$ \\
\hline & $\begin{array}{l}\text { método de } \\
\text { detecção }\end{array}$ & 008 & \\
\hline
\end{tabular}

Figura 1: O modelo de dados OntoDomo: a entrada conceito

\title{
2.1. A Entrada Conceito
}

Para além do subdomínio, a entrada conceito inclui os seguintes elementos (Figura 1):

\section{a) A designação (normalizada) do conceito (label)}

A designação do conceito emerge da descrição do corpus, segundo uma metodologia ascendente. Da aquisição de unidades terminológicas e relações conceptuais, resulta um conjunto de unidades linguísticas designando o mesmo conceito, as quais, depois de estruturadas, foram validadas pelos especialistas, formando um paradigma linguístico, no exemplo, com os seguintes elementos:

\author{
detector de gás \\ detectores de gás \\ detector de fuga de gás \\ detectores de fuga de gás \\ detector de fugas de gás \\ detectores de fugas de gás \\ sensor de gás \\ sensores de gás \\ sensor de fuga de gás \\ sensores de fuga de gás
}


Em seguida, foi estabelecida, pelos especialistas, a cota de aceitabilidade terminológica (term acceptabily rating) segundo a classificação definida pelas normas ISO (cf. ISO 107-1:2000: 8):

$$
\begin{aligned}
& \text { TP - termo privilegiado (preferred term); } \\
& \text { TA - termo admitido - tolerado (admited term); } \\
& \text { TD - termo desaconselhado (deprecated term); } \\
& \text { TO - termo obsoleto (obsolete term). }
\end{aligned}
$$

No exemplo, detector de gás é assumido como o termo privilegiado (TP) e, consequentemente, é adoptado como designação (label) do respectivo conceito, partilhando a atribuição de um mesmo identificador numérico, neste caso, 82.00.

b) A explicitação das relações genéricas

A relação conceptual genérica, is_a, relaciona cada conceito subordinado com o respectivo conceito genérico. A relação conceptual genérica constitui o travejamento de qualquer recurso ontológico baseado nos mecanismos da herança (cf. Bourigault e Aussenac-Gilles, 2004: 31).

is_a (“detector de gás", “detector”).

c) A explicitação das relaçōes partitivas

Neste modelo, de acordo com a nossa pesquisa, são utilizadas duas das categorias da relação partitiva da taxinomia de Winston et al. (1987), consideradas mais relevantes, no domínio: a relação componente-objecto (part_of_c), e a relação fase-processo (part_of_ph).

d) A explicitação das relações funcionais

Entre as relações funcionais, «télicas», que têm sido objecto de aprofundada reflexão por parte de muitos autores como Pustejovsky et al. (2006) e, e.g., Vossen (2006) e Lautenbacher (2000), utilizámos dois níveis de funcionalidade: a função imediata (used_for) e as finalidades genéricas do um sistema domótico (has_purpose).

e) Explicitação das relações especifficas

Relativamente às relações específicas do domínio, sendo em grande número, limitámo-nos, neste estudo, ao tratamento, exemplificativo, de 
algumas, que permitem a definição diferencial de sistemas conceptuais modelizados, como os de sensor e detector. Deve anotar-se que as aplicações de pesquisa, baseiam-se, essencialmente, na relação genérica (is_a), afastando-se da tendência formal das ontologias e bases de conhecimentos, produzidas no âmbito da inteligência artificial.

\subsection{A Entrada Termo}

Como se explicita na figura 2, a componente linguística da base de conhecimentos terminológicos inclui a seguinte informação:

- A unidade terminológica (UT), designadora do conceito (termo privilegiado - TP), com o mesmo identificador numérico do conceito (82.00);

- Um exemplo (contexto) significativo, do uso da UT, no corpus;

\begin{tabular}{|c|c|c|}
\hline UT & 82.00 & DETECTOR DE GÁS \\
\hline Categoria gramatical & 01 & s. $\mathrm{m}$. \\
\hline $\begin{array}{l}\text { Definição terminográfica } \\
\text { gerada }\end{array}$ & & $\begin{array}{l}\text { Detector } \\
\text { Entrada - presença de gás doméstico (butano, propano ou gás natural) } \\
\text { Saída - sinal eléctrico binário }(0 / 1) \\
\text { Usado para - deteç̧ão de fugas de gás } \\
\text { Objectivo - protecção contra incêndios }\end{array}$ \\
\hline Exemplo & & $\begin{array}{l}\text { [...] em caso de fuga de gás os detectores de gás permitem ao sistema actuar } \\
\text { e fechar as electroválvulas de entrada de gás. } \\
\text { Manuel SEVERIANO et al ( } 2005) \\
\text { Page: } 58 \mathrm{j} \text { (1e occ.) }\end{array}$ \\
\hline $\begin{array}{l}\text { UT, sinónimos e variantes } \\
\text { do corpus }\end{array}$ & $\begin{array}{l}82.00 \\
82.01 \\
82.02 \\
82.03 \\
82.04\end{array}$ & $\begin{array}{l}\text { detector de gás } \\
\text { detectores de gás } \\
\text { detector de fuga de gás } \\
\text { detectores de fuga de gás } \\
\text { detector de fugas de gás } \\
\text { detectores de fugas de gás } \\
\text { sensor de gás } \\
\text { sensores de gás } \\
\text { sensor de fuga de gás } \\
\text { sensores de fuga de gás }\end{array}$ \\
\hline \multicolumn{3}{|l|}{ Variantes abreviadas } \\
\hline Equivalente francês & & détecteur de gaz \\
\hline Equivalente inglês & & gas detector \\
\hline NOTAS & & \\
\hline
\end{tabular}

Figura 2: O modelo de dados OntoDomo: a entrada termo 
- O conjunto dos termos sinónimos e suas variantes, no plano da expressão. As unidades sinónimas tomam os identificadores numéricos, incrementados em uma unidade, a partir do identificador da UT privilegiada: $82.01,82.02,82.03$ e 82.04, tendo em vista a facilitação do tratamento informático. No exemplo de detector de gás, todos os termos sinónimos foram validados e classificados segundo a norma ISO (ISO 107-1:2000: 8). São também incluídas as variantes flexionais de número (plural).

- Variantes abreviadas (siglas e acrónimos);

- Termos equivalentes nas línguas inglesa e francesa;

- Um campo "NOTAS".

Não existindo, como é reconhecido, praticamente, sinónimos absolutos, no plano do significado linguístico, sempre que a comutação dos termos sinónimos não altere o valor de verdade das proposições registadas no corpus, os termos são agrupados numa única entrada terminológica, sendo as restantes consideradas variantes, do plano da expressão, evitando-se, deste modo, a redundância resultante da multiplicação de "fichas terminológicas" desnecessárias. Realizado todo o trabalho de descrição do corpus, na fase de normalização linguística, a análise das propriedades dos objectos, quando possível, será também um critério a ter em conta (cf. Depecker, 2005: 11).

Uma lista alfabética de todos os termos, permitirá o acesso à entrada terminológica em que figura cada um dos termos, bem como à respectiva entrada conceito.

\section{A multifuncionalidade da aplicação ontodomo}

Não cabendo nestas páginas, por falta de espaço, uma descrição da arquitectura complexa do sistema OntoDomo, desenvolvido a par da presente investigação, no Centro de Linguística da Universidade Nova de Lisboa, importa referir que, em relação à interface com o utilizador (figura 3), a aplicação OntoDomo apresenta-se, no plano ergonómico, adequada ao utilizador não linguista / terminólogo, pensada, especialmente, para estudantes da área de domótica. 


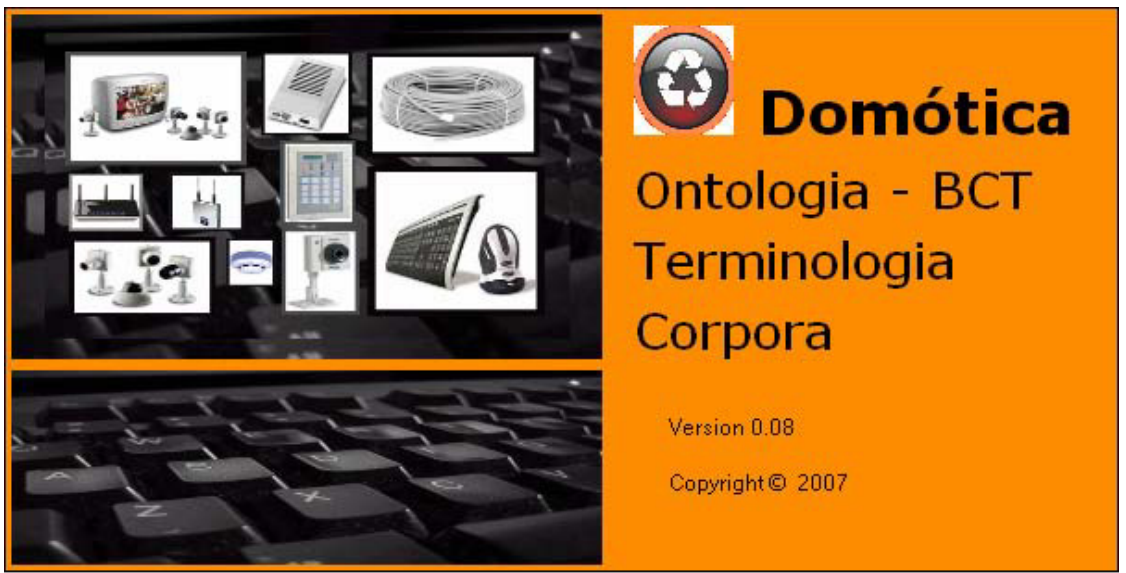

Figura 3: Ecrã de entrada da aplicação OntoDomo

Os utilizadores do sistema OntoDomo beneficiam do trabalho minucioso de análise linguística de corpora e do seu tratamento por especialistas de reconhecido prestígio, do domínio de edifícios inteligentes e domótica e, da área da engenharia do conbecimento.

\subsection{Ontologia e Base de Conhecimentos Terminológicos: Edição e Navegação}

O núcleo conceptual de uma base de conhecimentos terminológicos é constituído por uma ontologia. A navegação, numa ontologia permite, no plano didáctico e da ergonomia cognitiva, visualizar a estrutura de um sistema conceptual, reduzindo a multiplicidade das expressões linguísticas a um "mapa cognitivo" facilitador da compreensão do domínio, por exemplo, do microssistema conceptual de detector. Como evidencia a figura 4, o sistema. 


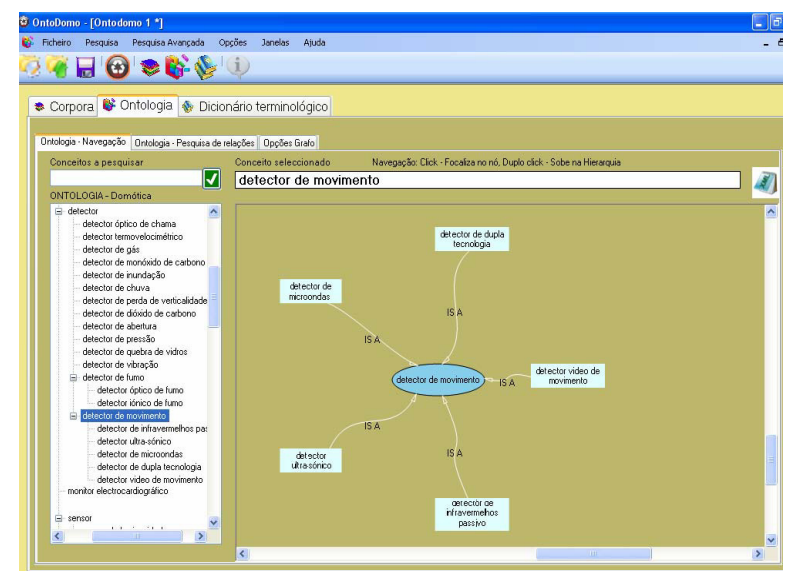

Figura 4: Navegação no núcleo ontológico do sistema conceptual de detector; visualização dos conceitos específicos de detector de movimento

OntoDomo permite que o utilizador se desloque ao longo da taxinomia e visualize, através da interface gráfica, as relações genéricas existentes entre os conceitos da ontologia, podendo, a partir de qualquer conceito desta "espinha dorsal", aceder aos pormenores da informação linguística contida na base de conhecimentos.

Neste modelo relacional, cada conceito define-se pelas relações que sustém em relação aos restantes, sendo a relação de especialização, (isa) o suporte estrutural das restantes relações.

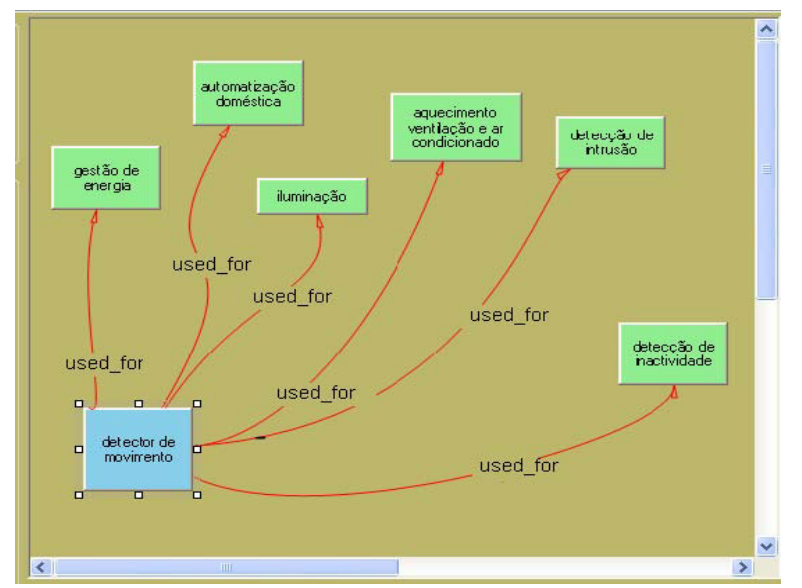

Figura 5: Grafo conceptual gerado pelo sistema OntoDomo, traduzindo o conjunto das asserções baseadas no predicado used_for 
$\mathrm{Na}$ figura 5, é representado um grafo conceptual, gerado pelo sistema OntoDomo, traduzindo as relações télicas do conceito detector de movimento, com os conceitos subordinados, específicos, de função domótica: detecção de intrusão, gestão de energia, AVAC, iluminação, automatização doméstica e deteçãao de inactividade.

Cada linha do grafo conceptual corresponde a uma asserção lógica, por exemplo, «used_for ("detector de movimento", "detecção de intrusão")».

Na figura 6, são explicitadas relações partitivas e genéricas (sistema misto de conceitos (cf. (ISO 704, 2000: 14), sendo de anotar a herança de propriedades de dispositivo domótico pelos respectivos conceitos específicos: dispositivo sensor

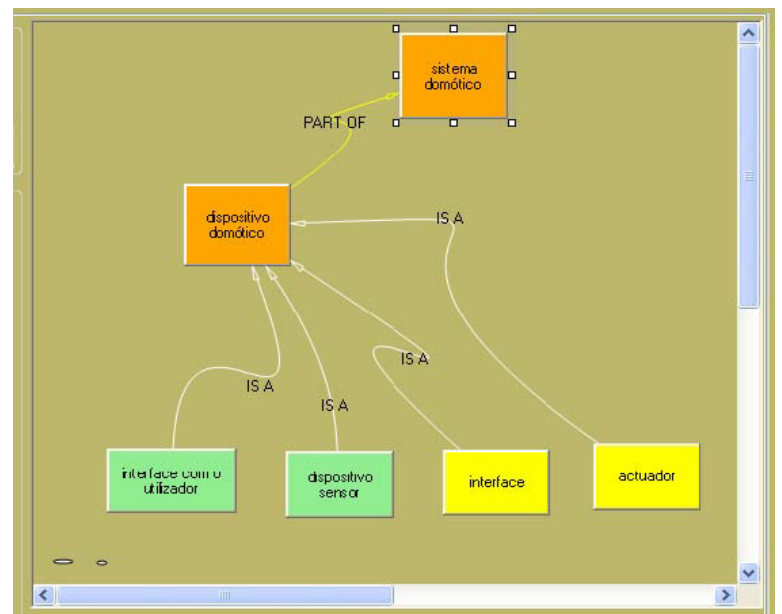

Figura 6:. Grafo conceptual gerado pelo sistema OntoDomo, representando um sistema misto de conceitos (relações is_a e part_of_c )

(sensores e detectores), actuador, interface e interface com o utilizador. Na figura 7 , está representada uma parte do suporte do conceito de dispositivo sensor, explicitados os conceitos subordinados de sensor: sensor biométrico, sensor de luminosidade, sensor de temperatura e sensor de velocidade do vento ou anemómetro. 


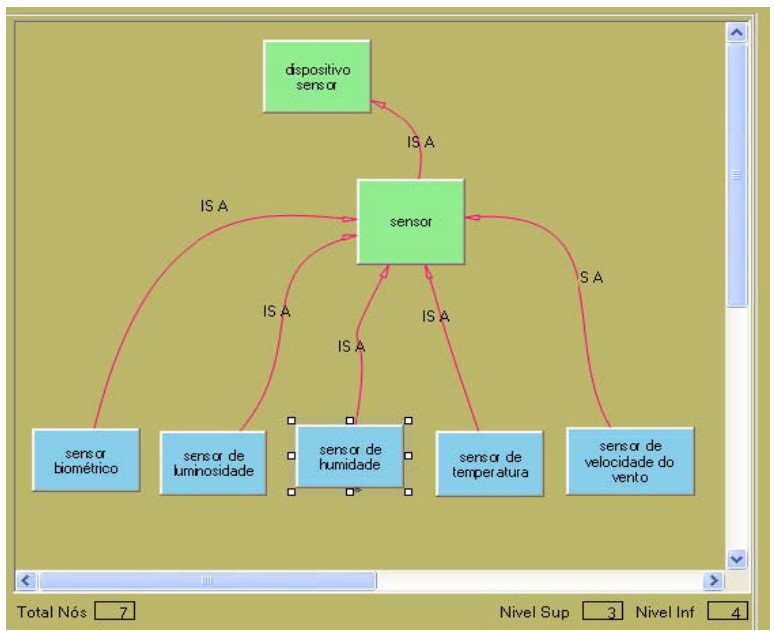

Figura 7: Suporte (parcial) da classe dispositivo sensor

\subsection{Visualização da Informação Terminológica Contida na BCT}

Como referido, a interface do OntoDomo, foi concebida para o utilizador não terminólogo, exibindo uma fich terminológica em formato convencional, a partir da base de conhecimentos (figura 8). Para além da categoria gramatical, do domínio, das variantes em língua francesa e inglesa, é apresentado um contexto considerado significativo e uma definição, em língua natural, explicitando as características do conceito. Trata-se de um ambiente próximo do de um "dicionário terminológico".

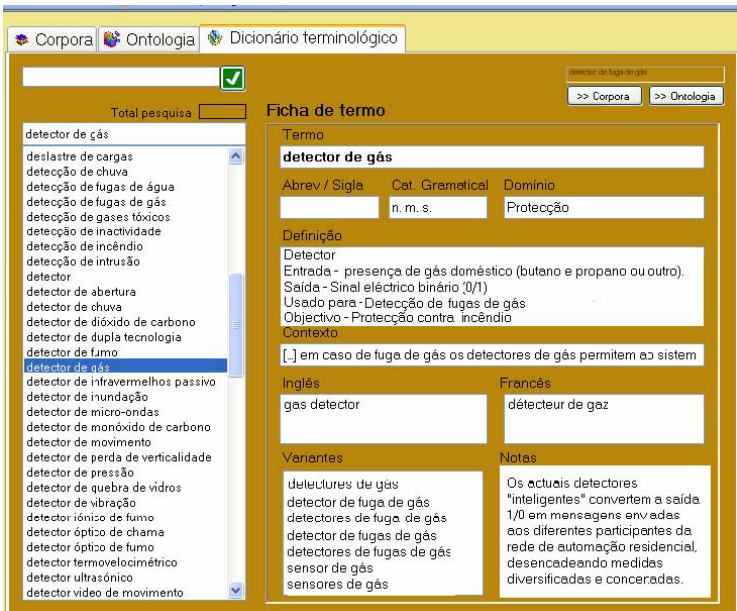

Figura 8: A exibição de conhecimentos terminológicos contidos na BCT 


\subsection{Um Motor de Pesquisa Assistida, Selectivo e Flexível}

Um dos problemas dos actuais motores de pesquisa, como instrumentos da investigação, é o ruído resultante do uso de formas simples, e da existência de múltiplas variantes que o utilizador ou não retém na memória, por serem, frequentemente, muito numerosas, ou pelo tempo necessário à sua introdução manual, na entrada do motor de pesquisa. O método tornase pouco eficaz, por excesso de ruído, ou de silêncio, representando grande desperdício de tempo, em particular, para o estudante inexperiente, que inicia um curso de especialização. Por outra parte, a selecção das palavras-chave não é devidamente instruída, resultando em sobrecarga cognitiva, defluente da obtenção de dados prolíferos, irrelevantes ou contraditórios. A presente investigação pretendeu dotar o sistema OntoDomo de conhecimentos suficientemente relevantes para que o mesmo possa constituir uma ferramenta verdadeiramente útil, na consulta de documentação especializada.

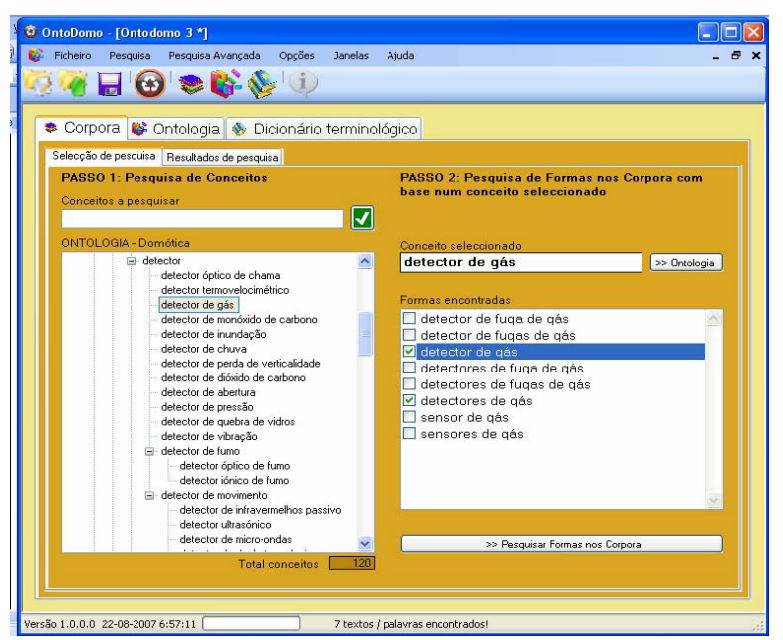

Figura 9: Pesquisa assistida, selectiva, a partir de um dos conceitos da ontologia

O princípio de funcionamento é ilustrado pela figura 9 e centra-se no núcleo conceptual da base de conhecimentos: a ontologia, a qual permite partir de uma síntese do domínio, cognitivamente acessível, para a diversidade das expressões linguísticas dos conceitos. Como ilustra a figura referida, o sistema OntoDomo propõe uma lista de termos ou variantes de um termo, bastando ao utilizador seleccionar todas ou apenas algumas das denominações sugeridas. Para o conceito detector de gás, o sistema propõe um conjunto de termos validados, extraídos do corpus Domo. 
Propõe também a designação privilegiada do conceito (detector de gás), estabelecida pelos especialistas, no processo da normalização linguística. Por esta via, evita-se o ruído resultante da utilização de termos genéricos e beneficia-se de um trabalho aturado de análise linguística e modelização conceptual, tutelado pelos especialistas do domínio.

Como foi referido, oportunamente, a variação terminológica é um fenómeno normal, generalizado e que merece o maior destaque, em particular, na pesquisa e consulta de documentação técnica, assumindo dimensões que foram, durante muito tempo, subestimadas (cf., e. g., Gaudin, 2003). A supervisão do especialista permitiu validar os dados introduzidos no sistema OntoDomo, tendo em conta que a observação minuciosa dos corpora suscita reflexão aprofundada, tanto no plano linguístico como no plano do domínio de especialização. Como exemplo, poderemos referir a substituição do termo detector de gás por sensor de gás, recolhendo o segundo cerca de $75 \%$ das ocorrências do conceito.

Observar o corpus e reflectir sobre os dados obtidos com os especialistas constitui um processo endógeno de valorização da língua portuguesa, como sistema modelizante da ciência e da tecnologia.

É por isso, oportuno que um sistema de pesquisa assistida possa oferecer alguma orientação aos estudantes que iniciam uma especialização. Esta também constitui uma valência funcional do modelo OntoDomo. Como se exemplifica através do tratamento do conceito sensor de luminosidade ou sensor de intensidade luminosa, o motor de pesquisa, para além de facultar as diversas designações, acrescenta informação visível sobre a classificação relativa às cotas de aceitabilidade terminológica (CA), definidas pela ISO, por forma a que o utilizador seja prevenido quanto à valorização atribuída às diferentes opções.

A figura 10 ilustra esta função pedagógica do módulo de pesquisa do OntoDomo, ao classificar as diferentes unidades terminológicas. Deste modo o termo privilegiado, sensor de luminosidade, foi seleccionado para label do conceito: opção que implicitamente lhe confere o estatuto de termo preferido. 


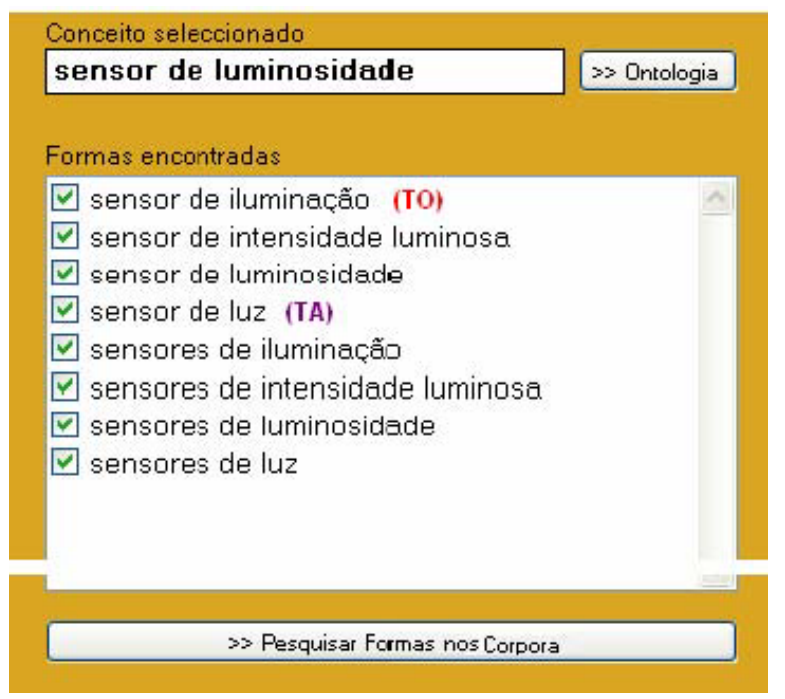

Figura 10: Proposta de pesquisa orientada através da indicação da cota de aceitabilidade terminológica (CA)

Dos restantes termos validados, um grupo apresenta-se normal, sem classificação, nem preferido nem desaconselhado. Os restantes são desvalorizados, sendo considerados termos tolerados, desaconselhados ou obsoletos. No exemplo, poderemos afirmar, com base no processo de validação, que tanto sensor de luminosidade como sensor de intensidade luminosa são termos valorizados, sendo quase arbitrária a selecção de um ou de outro como label do conceito. Em relação às designações sensor de iluminação e sensor de $\mathrm{W}_{2}$ a primeira foi classificada como termo obsoleto (TO); a segunda como termo admitido / tolerado (TA).

Esta informação de carácter normativo figura na base de conhecimentos terminológicos. Tratando-se de um corpus essencialmente académico, em geral, já passou pelo processo de avaliação académica. Por isso, são em número muito reduzido os termos não validados pelos especialistas. Em relação ao conceito sensor de luminosidade, foi eliminado, no processo de validação, o candidato a termo sensor de iluminância, sendo, por isso, excluído na base de conhecimentos não sendo, subsequentemente, proposto pelo sistema OntoDomo.

Um sistema de pesquisa deve ser eficaz, o que passa também pelo tratamento dos termos equivalentes do inglês, "importados" com a própria tecnologia. Um exemplo elucidativo provém da tecnologia domótica euro- 
peia com maior implantação: a tecnologia EIB (European Installation Bus). Sendo uma tecnologia não proprietária (aberta), faculta e divulga as especificações técnicas, em várias línguas.

O termo unidade de acoplamento ao barramento, seria a tradução mais fiel de bus coupling unit (BCU). Contudo, no domínio da informática, bus constitui um empréstimo, sinónimo de barramento (cf. ILTEC: 1993). Assim, coexistem, no corpus Domo, como designações equivalentes a bus coupling unit, o termo acima referido e a denominação, mais curta, acoplador de bus. Contudo, o termo mais utilizado é a variante abreviada de bus coupling unit: $B C U$. Tendo em conta que a designação mais utilizada, no corpus é a sigla $B C U$, a mesma não pode deixar de figurar, como entrada essencial, do motor de pesquisa, como se ilustra na figura11. Com efeito, o trabalho terminológico,

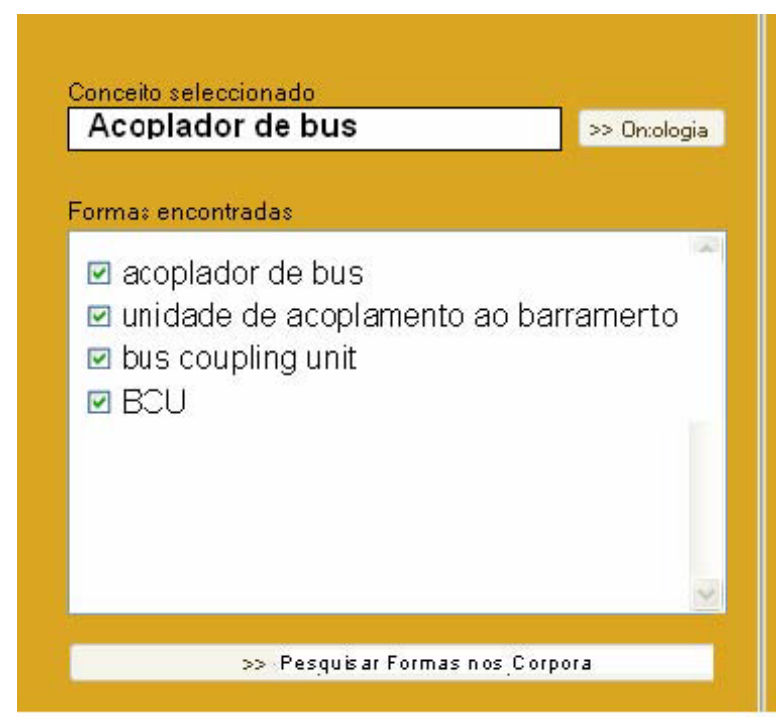

Figura 11: A inclusão de termos equivalentes

quando orientado para a recuperação de informação e consulta de documentação especializada, não pode prescindir dos meios linguísticos que conduzem às ocorrências dos conceitos, no corpus.

O termo equivalente não abreviado inglês, bus coupling unit, co-ocorre, quase sempre, com a sigla $B C U$ e apresenta uma frequência reduzida, em contexto parentético, servindo para explicar a leitura da sigla: 


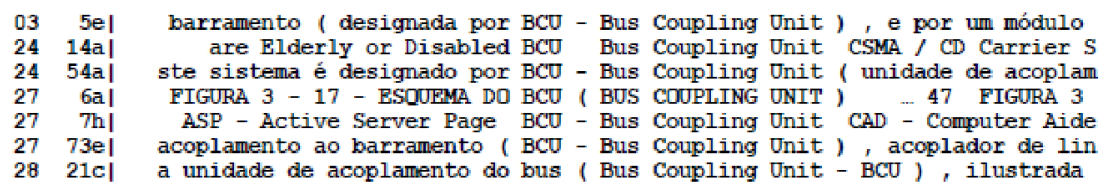

O mesmo não acontece com a sua abreviação, $B C U$, a qual apresenta um número de ocorrências tão elevado, no corpus Domo, que deve ser considerada um empréstimo, necessário à pesquisa, no corpus, como se infere da figura 12,

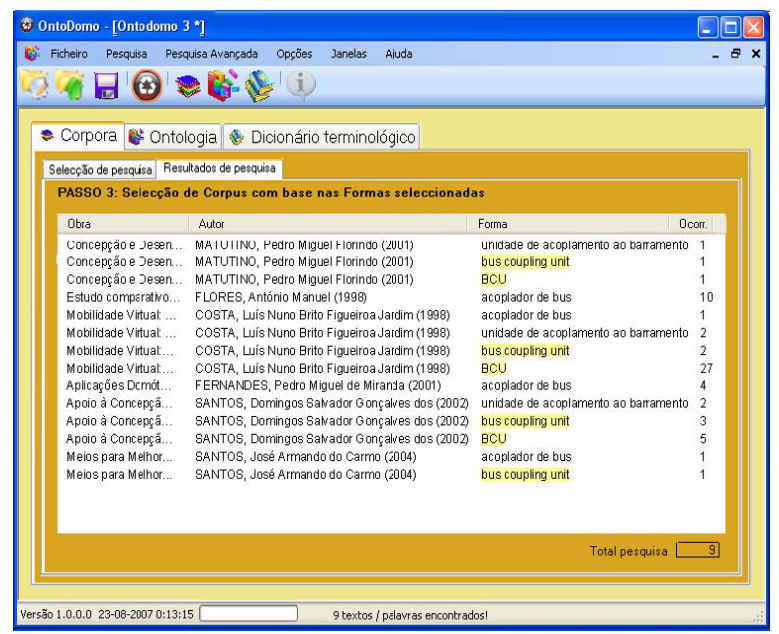

Figura 12: A primeira fase de acesso ao corpus, pelo sistema OntoDomo: o resumo dos dados

que sumaria os resultados da pesquisa solicitada na janela de entrada do OntoDomo. Observando os resultados facultados pelo OntoDomo, poderemos extrair as primeiras hipóteses para a prossecução da pesquisa, com base nas frequências:

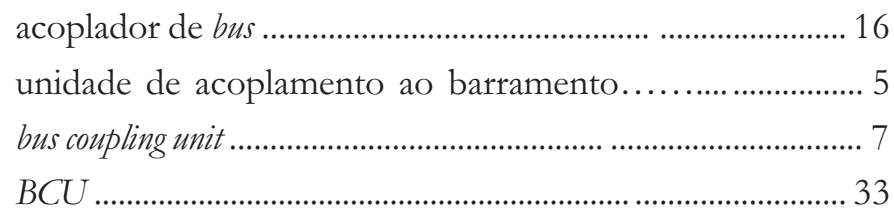

No exemplo de bus coupling unit, verificamos que o termo não abreviado, co-ocorre sempre com a sigla $B C U$, não servindo, portanto, para recu- 
perar mais informação. A informação que recupera é parcialmente redundante. $\mathrm{O}$ mesmo não acontece com a sigla $B C U$, indispensável ao motor de pesquisa, como revela a consulta em texto integral, facultada pelo OntoDomo. De resto, nos textos académicos, é frequente encontrar segmentos discursivos como o que sublinhámos na saída do Ontodomo:

A unidade de acoplamento ao barramento, doravante designada por BCU, é parte integrante de $[\ldots]$

O que é peculiar, neste caso, é que a sigla BCU não corresponde ao termo não reduzido, unidade de acoplamento ao barramento, mas ao termo do inglês bus coupling unit.

Como pode observar-se, nos resultados da aplicação Ontodomo, todos os termos da pesquisa são, de imediato, assinalados a sombreado colorido. A forma particular da pesquisa, neste exemplo, unidade de acoplamento ao barramento, surge assinalada com uma cor mais escura.

A figura 13 representa o resultado de um experimento, ainda não completamente implementado no OntoDomo. Na versão actual, já estabilizada, apenas são propostas pela aplicação, para pesquisa, os termos em língua portuguesa e os empréstimos do inglês. Contudo, dada a convivência "indecisa", frequente, de termos de língua portuguesa e inglesa, os equivalentes do inglês poderão vir a constituir também "variantes" a ter em conta, na instrução do motor de pesquisa.

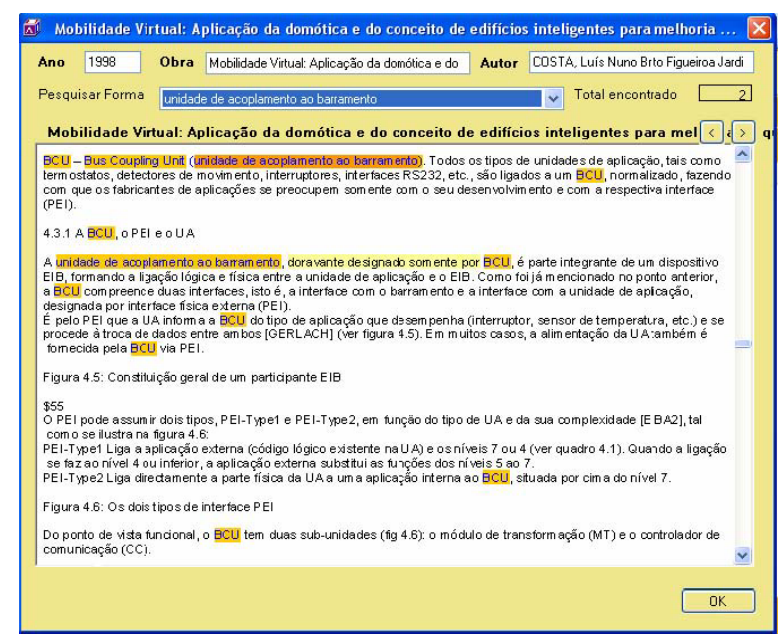

Figura 13: Os resultados da pesquisa assistida pelo OntoDomo do conceito acoplador de bus 
Para além das principais funcionalidades já exemplificadas, o sistema OntoDomo permite também projectar no corpus todas as unidades terminológicas e suas variantes, assinalando (a cor azul) todos os termos do corpus que se encontram na base de conhecimentos, conforme ilustra a figura 14 .

Este tratamento dos textos é, em si mesmo, uma forma de assistir a consulta (leitura) dos documentos. O nosso modelo de BCT permite, por esta via, a indexação automática, uma vez que a BCT forma, como o seu núcleo, a ontologia, um indice estruturado. Contudo, esta função ainda não foi implementada no sistema OntoDomo.

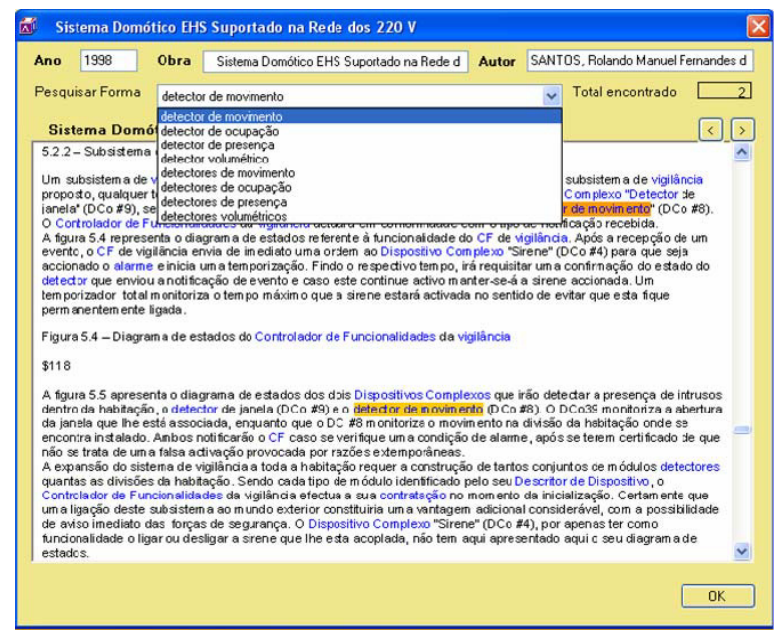

Figura 14: A projecção nos textos do corpus da terminologia do domínio

No estado actual de desenvolvimento, esta aplicação constitui, essencialmente, uma ferramenta para consulta assistida de documentação especializada no domínio da domótica. Para terminar estas referências à aplicação OntoDomo, cabe referir que a mesma constitui um sistema hipertextual avançado, potenciando a navegação entre a multiplicidade de elementos que articula, pertencentes aos corpora è̀ ontologia / BCT.

\section{Referências bibliográficas}

BACHIMONT, B. 2005. Corpus et connaissances: De l'extraction linguistique à la modélisation conceptuelle. In : CONDAMINES, A. (éd.). Sémantique et corpus. Paris: Hermès Science. p. 319-46. 
BANEYX, A. et al. 2005. Synergie entre analyse distributionnelle et patrons lexico-syntaxiques pour la construction d'ontologies différentielles. In : Actes de la Conférence TLA 2005 (Gèmes Rencontres Terminologie et Intelligence Artificielle) Rouen: Université de Rouen, em linha, http:/ /www. loria.fr/ yannick/TIA2005/doc/, consultado em 09/ 08/2005.

BENTO, J. R. 2007. Da constituição do corpus à construção de uma ontologia e base de conhecimentos terminológicos. Dissertação (Doutoramento em Linguística / Lexicologia). Universidade Nova de Lisboa.

BIÉBOW, B.; SZULMAN, S. 2000. Une approche terminologique pour catégoriser les concepts d'une ontologie. In: CHARLET, J.; ZACKLAD, M.; KASSEL, G.; BOURIGAULT, D. (éd.). Ingénierie des connaissances: évolutions récents et nouveaux défis. Paris: Eyrolles, p. 325-36.

BOURIGAULT, D. 2002. Upery: Un outil d'analyse distributionnelle étendue pour la construction d'ontologies à partir de corpus. In: Actes de la 9ème Conférence Annuelle sur le Traitement Automatique des Langues (TALN 2002). p. 75-84.

; CHARLET, J. 2000. Ontologies et textes. In : Actes des 11èmes Journées Ingénierie des Connaissances (IC'2000). Toulouse: Centre pour l'Unesco, p. 7-8, em linha, http://www.irit.fr.ic 2000, consultado em 03/04/2003.

; AUSSENAC-GILlES, N.; CHARLET, J. 2004. Construction de ressources terminologiques ou ontologiques à partir de textes: un cadre unificateur pour trois études de cas. Revue des Sciences et Technologies de l'Information (RSTI) série: Revue d'intelligence artificielle (RIA) 18, n. ${ }^{\circ}$ 1. 87-110.

; JACQUEMIN, C.; L'HOMME, M. C. 2001. Recent advances in computational terminology. Amsterdam/ Philadelphia: John Benjamins.

BUSA, F.; CALZOLARI, N.; LENCI, A. 2001. Generative lexicon and the SIMPLE model: Developing semantic resources for NLP. In: BUSA, F.; BOUILLON, P. (ed.). The language of word meaning. Cambridge: Cambridge University Press. p. 333-49.

CABRÉ, M. T.; FELIU, J.; VIVALDI, J. 2004. Base de connaissances GENOMA: Le rôle de l'ontologie. Workshop on Terminology, Ontology and Knowledge représentation (TERMINO 2004). Lyon: Université Jean-Moulin Lyon 3. p. 19-25.

CHEIN, M.; MUGNIER, M. 1992. Conceptual graphs: fundamental notions. Revue d'Intelligence Artificielle 6, n. ${ }^{\circ}$ 4. 365-406.

CHINGAREVA-SLAVINE, E. 2003. Sémiotique linguistique et modélisation. Lavoisier: Hermès Science.

CONDAMINES, A. 2003. Sémantique et corpus spécialisés: Constitution de bases de connaissances terminologiques. Mémoire d'Habilitation à Diriger les Recherches. Toulouse: Universidade Toulouse 2 .

CONDAMINES, A. (éd.). 2005a. Sémantique et corpus. Lavoisier: Hermès Science.

CORBIN, D. 1987. Morphologie dérivationnelle er structuration du lexique. 2 vols. Tubinga: Max Niemeyer Verlag.

CORCHO, O. et al. 2003. Methodologies, tools and languages for building ontologies. Where is their meeting point?. Data \& Knowledge Engeneering 46. 41-64.

CRUSE, D. A. 2000. Meaning in Language. An introduction to Semantics and Pragmatics. Nova York: Oxford University Press.

DEPECKER, L. 2005. Contribution de la terminologie à la linguistique. Langages 157. La terminologie: Nature et enjeux. 6-13. 
FELIU, J.; VIVALDI, J.; CABRÉ, M. T. 2002b. Towards an ontology for a human genome knowledge base. Third International Conference on Language Resources and Evaluation - LREC 2002. Las Palmas: ELRA, p. 1885-1890, também disponível em linha, https://nats www.informatik.unihamburg.de/intern/proceedings/2002/ LREC/htm/ papers.htm.

FRADIN, B. 2003. Nowvelles approches en morphologie. Paris: PUF.

GAUDIN, F. 2003. Socioterminologie: une approche sociolinguistique de la terminologie. Bruxelles: Duculot.

GÓMEZ-PÉREZ, A. 1999. Développements récents en matière de conception, de maintenance et d'utilisation des ontologies. Terminologies nouvelles 19. 9-20.

ISO 1087-1 2000-E/F. Terminology - Vocabulary. Part 1: Theory and apllication. Travaux terminologiques. Vocabulaire Partie 1: Théorie et application. Genève: Organisation Internationale de Normalisation.

L'HOMME, M. 2002. Fonctions lexicales pour représenter les relations sémantiques entre termes. Traitement automatique des langues 43, n. ${ }^{\circ}$ 1. 19-41

LAUTENBACHER, O. 2000. Les fondements perceptuels de la production de sens dans le lexique génératif. Thèse (Doctorat). Université Marc Bloch de Strasbourg.

LINO, M. T.; MEDINA, D.; MOREIRA, J. D.; CHICUNA, A. 2007. Rede de Neologia e de Terminologia em Língua Portuguesa (em situação de contacto de línguas).In: XVII encontro da Associação das Universidades de Lingua Portuguesa. AULP, Praia - Cabo Verde (no prelo). (éd.). 2003. Mots et lexiculture, hommage à Robert Galisson. Paris: Champion.

MARRAFA, P. 2002. Portuguese WordNet: general architecture and internal semantic relations. DELTA 18. 131-46.

MEYER, I. 2001. Extracting knowledge-rich contexts for terminography: a conceptual and methodological framework. In: BOURIGAULT, D.; JACQUEMIN, C.; L'HOMME, M. (ed.). Recent advances in computational terminology. Amsterdam/ Philadelphia: John Benjamins. p. 279302.

PUSTEJOVSKY, J. et al. 2006. Towards a generative lexical resource: the brandeis semantic ontology. In: CALZOLARI, N.; CHOUKRI, K.; GANGEMI, A.; MAEGAARD, B.; MARIANI, J.; ODJIK, J.; TAPIAS, D. (ed.). In: Proceedings of the 5th International Conference on Language Resources and Evaluation. LREC 2006, Génova: ELRA, p. 1702-05.

SOWA, J. F. 2000. Knowledge representation: logical, philosophical, and computational foundations. New York: Brooks Cole Publishing.

VOSSEN, P. 2006. EuroWordNet general document, University of Amsterdam, em linha, http:/ /www.hum.uva.nl/ ewn, consultado em 10/10/2008.

WINSTON, M. E.; CHAFFIN, R.; HERRMANN, D. 1987. A taxonomy of part-whole relations. Cognitive Science 11, n. ${ }^{\circ}$ 4. 417-44.

XAVIER, M. F.; MATEUS, M. H. M. (ed.). 1990. Dicionário de termos linguísticos. v. I. Lisboa: Edições Cosmos. 